\title{
Estimation of the fibre temperature during the inscription of arc-induced long-period gratings
}

\author{
Gaspar M. Rego ${ }^{\text {a,b,* }}$, Paulo V.S. Marques ${ }^{\text {b,c }}$, José L. Santos ${ }^{\text {b,c }}$, Henrique M. Salgado b,d \\ ${ }^{a}$ Escola Superior de Tecnologia e Gestão, IPVC, Av. do Atlântico, 4900-348 Viana do Castelo, Portugal \\ ${ }^{\mathrm{b}}$ UOSE, INESC-Porto, Rua do Campo Alegre 687, 4169-007 Porto, Portugal

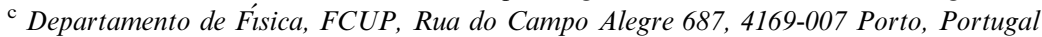 \\ ${ }^{\mathrm{d}}$ Departamento de Engenharia Electrotécnica e de Computadores, FEUP, Rua Dr. Roberto Frias, 4200-465 Porto, Portugal
}

Received 5 July 2005; received in revised form 11 September 2005; accepted 12 September 2005

\begin{abstract}
The underlying formation mechanisms and the properties of long-period gratings produced through arc discharges are intrinsically related to the temperature reached by the fibre during arc exposure. In this work, the determination of the fibre temperature was based on Plank's blackbody radiation law. The radiation emitted by the optical fibre during heating due to an electric arc discharge, detected using a Cronin spectrometer, was fitted to the emission spectrum of the blackbody radiation, allowing the estimation of the temperature range attained by the fibre. A peak temperature of $1400 \pm 50{ }^{\circ} \mathrm{C}$ was obtained.
\end{abstract}

(c) 2005 Elsevier B.V. All rights reserved.

PACS: $42.81 . \mathrm{Wg}$

Keywords: Temperature measurement; Blackbody radiation; Electric arc discharge; Arc-induced gratings; Long-period gratings; Fibre gratings

\section{Introduction}

Long-period fibre gratings (LPFGs) are periodic structures, typically with periodicities of hundreds of microns, which couple light from guided to cladding modes at specific wavelengths that satisfy the resonance condition [1]. LPFGs transmission spectra are characterized by dips at those particular wavelengths which, in turn, depend on physical parameters such as temperature and pressure. Therefore, these gratings are wavelength selective tunable filters that find a wide range of application as optical communications devices and as optical sensors [2]. In the latter domain, arc-induced LPFGs and UV-induced fibre Bragg gratings (FBGs) have been used for the simultaneous measurement of temperature and strain [3]. The search for new applications or optimization of these

\footnotetext{
* Corresponding author. Tel.: +351226082 601; fax: +351226082699. E-mail address: gmrego@fc.up.pt (G.M. Rego).
}

devices demands a detailed understanding of the physical mechanisms that determine their characteristics. In the case of UV written LPFGs and FBGs, the underlying physical principle is the photosensitivity of silica waveguides, which has been the subject of extensive research. Arc-induced long period gratings proved already to be an interesting alternative to the devices fabricated by UV radiation [4]. In this context, the determination of the temperature reached by the fibre during the electric arc discharge is a key parameter in order to control adequately the fabrication of these structures.

In this paper, we present a method based on the blackbody radiation to determine the temperature of the fibre during electric arc exposure.

\section{Methodology}

The fibre temperature during an arc discharge is measured through the fitting of the blackbody expression to 
the radiation emitted by the fibre and detected using a Cronin spectrometer. The low intensity radiation emitted by the fibre section after being heated by an arc discharge of short duration ( $1 \mathrm{~s})$ is guided through the same fibre towards the spectrometer where its spectral content is separated by a holographic grating being afterwards detected by an array of detectors. Note that each wavelength range is allocated simultaneously to a particular detector (there is no wavelength sweep). It should be stressed that an optical spectrum analyzer (OSA) is not suitable for such kind of measurements requiring high sensitivity and fast data acquisition. Moreover, a commercial spectrometer, such as, the USB 2000 from Ocean Optics with the appropriate grating could be used to perform these measurements but, instead we have used the Cronin spectrometer (a former prototype). Therefore, to estimate the fibre temperature several intermediate steps are required. First, the spectral response of the spectrometer has to be determined. Second, the method has to be tested by applying it to the measurement of well known predefined temperatures. Third, the fibre refractive index has to be determined since the radiation is generated inside a medium different from vacuum. Finally, the dependence of the fibre emissivity on temperature and wavelength, for the specified fitting range, has to be known.

The basis of this method is the Plank's blackbody radiation law [5], given by

$I(\lambda, T)=\frac{c_{1}}{n^{2} \lambda^{5}} \frac{1}{\mathrm{e}^{c_{2} / n \lambda T}-1}$,

where $c_{1}$ and $c_{2}$ are constants with values equal to $3.7415 \times 10^{-16} \mathrm{~W} / \mathrm{m}^{2}$ and $1.4388 \times 10^{-2} \mathrm{~m} \mathrm{~K}$, respectively. The other parameters have their common meaning.

Fig. 1 shows the blackbody emission spectra in vacuum and in silica for two different temperatures. As it can be seen, the increase of the refractive index or temperature displaces the spectrum towards shorter wavelengths and increases its intensity.

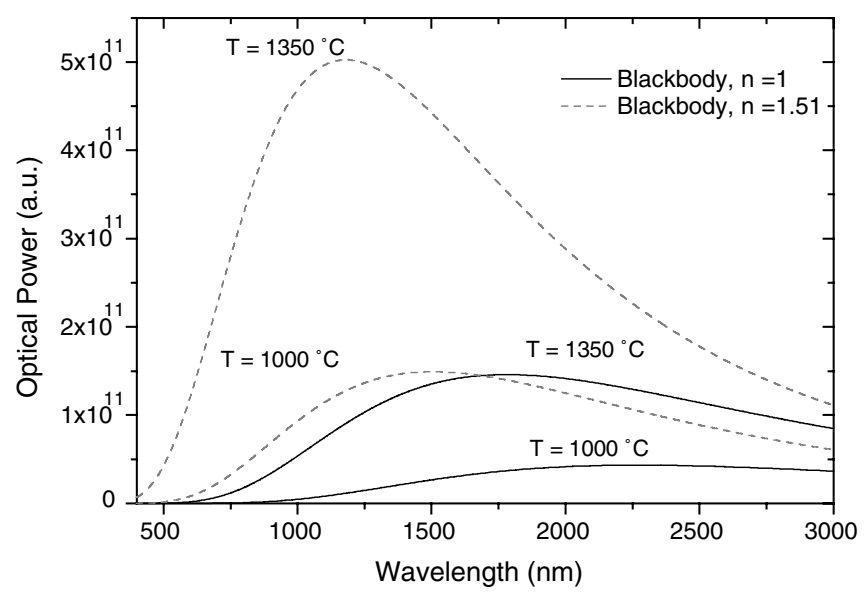

Fig. 1. Blackbody radiation for different temperatures and refractive indices.

\section{Results and discussion}

\subsection{Spectrometer spectral response}

The Cronin spectrometer consists of a holographic diffraction grating and an array of detectors which enables detection over the $310-1100 \mathrm{~nm}$ wavelength range, with an optical resolution of $10 \mathrm{~nm}$. A superluminescent laser diode with a peak wavelength at $846 \mathrm{~nm}$ and a bandwidth at FWHM of $29 \mathrm{~nm}$ was used to determine the spectrometer wavelength accuracy, Fig. 2. The spectrometer broad spectral response (Fig. 3) was calibrated by comparing the spectra of a white light source registered by the Cronin spectrometer and by a calibrated OSA. It is interesting to note that the spectral response of the Cronin spectrometer is similar to the commercially available USB 2000 from Ocean Optics using grating \#3.

\subsection{Validating the method}

Two pieces of multimode fibre ( $\sim 2 \mathrm{~m}$ long) were used to collect the radiation from an oven at different temperatures

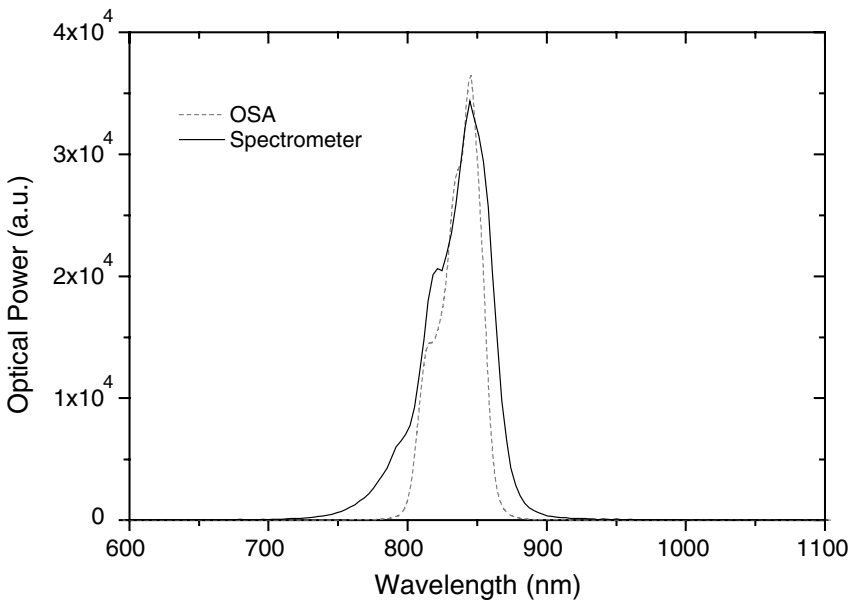

Fig. 2. Spectrometer wavelength accuracy.

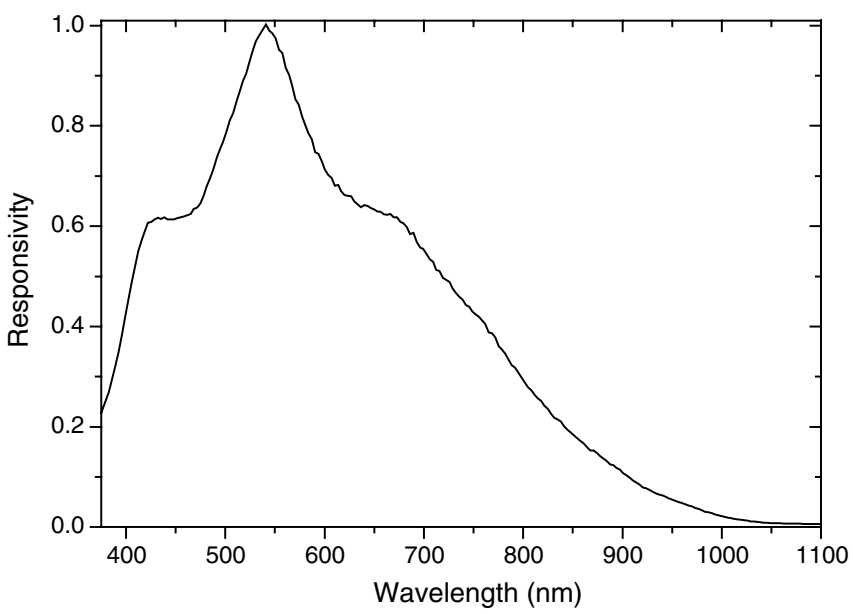

Fig. 3. Spectrometer responsivity. 
above $1000{ }^{\circ} \mathrm{C}$. One piece was connected to the OSA and the other one to the computer controlled spectrometer (Fig. 4). The recorded optical spectra were then fitted to the blackbody radiation expression in the $400-900 \mathrm{~nm}$ range. Note that in this case the value of $n$ was set equal to 1 since the optical fibre was only used to guide the light. Fig. 5 shows the emission spectra of the oven at temperatures above $1000{ }^{\circ} \mathrm{C}$, as measured by the OSA. The fitting of blackbody radiation expression to the emission spectrum corresponding to an oven temperature of $1350^{\circ} \mathrm{C}$ is also shown ( $c$ is an experimental constant resulting from fitting's optimization). The relative error in the determination of the oven temperature ( $\left.T_{\text {oven }}\right)$ was lower than $2 \%$, as shown in Fig. 6 for two (\#1 and \#2) separate measurements performed by using the OSA (circles) and the spectrometer (squares).

\subsection{Fibre refractive index}

To determine the refractive index of the fibre, a short length was placed inside an oven with one end connected to the OSA and the other one connected to the spectrometer, allowing simultaneous detection by both devices. The temperature of the oven was increased slowly up to $1350^{\circ} \mathrm{C}$. In this case, the radiation detected corresponds to the emission of the fibre while in thermal equilibrium with the oven. Fitting the blackbody radiation expression (450-900 $\mathrm{nm}$ range) to the spectra of several independent experiments resulted in a value of $1.48 \pm 0.03$ for the fibre refractive index. Taking into account that the refractive

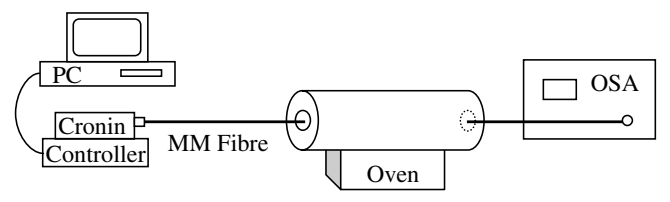

Fig. 4. Diagram of the set up used.

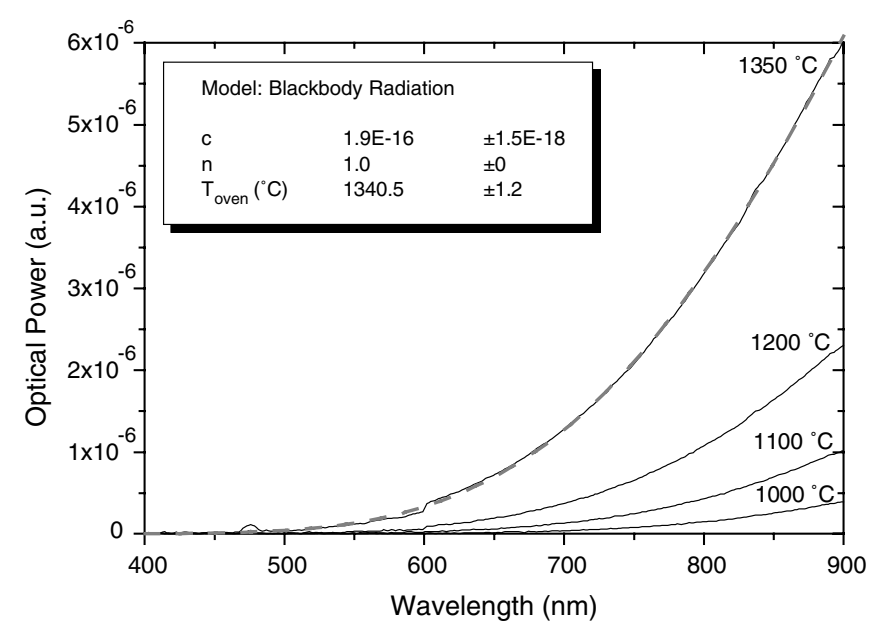

Fig. 5. Fitting blackbody radiation expression to the oven emission spectra, as detected by the OSA ( $c$ is an experimental constant and $T_{\text {oven }}$ is the temperature of the oven as determined from the fitting process).

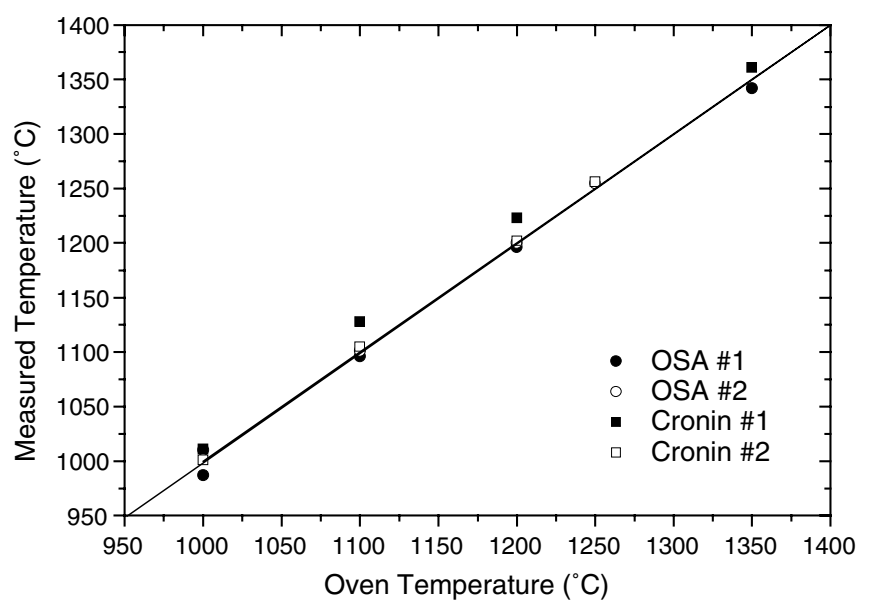

Fig. 6. Measured temperature using Plank's law versus oven temperature. Two sets (\#1 and \#2) of independent measurements are shown in the figure.

index of pure silica at room temperature is $1.456(675 \mathrm{~nm})$, that the core to cladding refractive index difference, $\Delta n$, is 0.012 and assuming that the temperature dependence of the refractive index $\mathrm{d} n / \mathrm{d} T$ is $\sim 2.5 \times 10^{-5}{ }^{\circ} \mathrm{C}^{-1}$ [6], the refractive index calculated for a temperature difference of $1330{ }^{\circ} \mathrm{C}$ is $\sim 1.50$. Note that the thermo-optic coefficient was estimated for temperatures up to $1000^{\circ} \mathrm{C}$. However, for higher temperatures, it is expected that this value falls due to a large increase of the silica thermal expansion coefficient $[6,7]$. Therefore, the refractive index value obtained experimentally agrees well with the one expected for the considered temperature. Finally, also note that the material dispersion for silica, obtained through Sellmeier's equation [8], has low influence on the temperature determination, being the difference within $10{ }^{\circ} \mathrm{C}$ (Fig. 7).

\subsection{Fibre emissivity}

To determine the fibre emissivity, the dependence of the silica based glass emissivity on wavelength and on temperature has to be known. It is also required knowledge of the geometry of the cavity and its respective

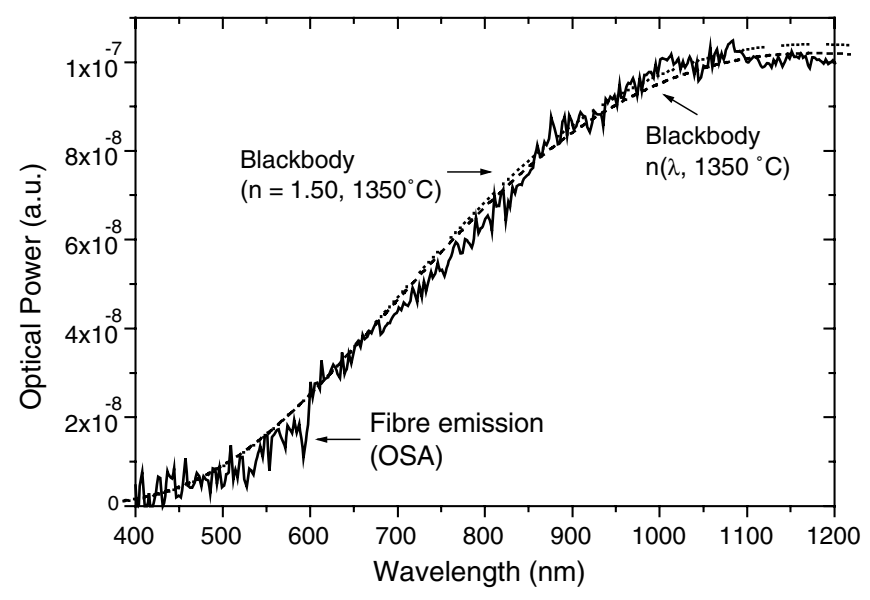

Fig. 7. Fibre emission. 
dimensions. As can be seen in Fig. 1, considering typical values for the fibre temperature the wavelength range of interest is between 0.4 and $3.0 \mu \mathrm{m}$. Note however that the spectral response of the spectrometer is limited to about $0.9 \mu \mathrm{m}$. Unfortunately, there is a lack of data regarding the emissivity of silica in this spectral range since it corresponds to a region of high transparency. The absorption is mainly due to the tail of the ultraviolet absorption band and therefore the respective absorption coefficient is relatively low, decreasing with increasing wavelength at room temperature [9]. However, recent results indicate a possible inversion of that dependence at high temperatures. In fact, it was observed that the absorption coefficient at $1.06 \mu \mathrm{m}$ is higher than at $0.5 \mu \mathrm{m}$ [10]. Regarding the temperature dependence, for the same wavelength range, it is known that the absorption increases rapidly for temperatures above $1050{ }^{\circ} \mathrm{C}$. These results were explained considering the formation of defect centres and due to electronic conduction [10].

Another important factor is the geometry of the cavity. For a cylindrical cavity it was shown [11] that its effective emissivity could be given by the following equation:

$\varepsilon_{\mathrm{eff}}=\varepsilon \frac{1+4 L / D}{1+\varepsilon(4 L / D)}$

where $\varepsilon$ is the emissivity of silica, $L$ is the length and $D$ the fibre diameter. For a fibre inside the oven, in which the length $(L=180 \mathrm{~mm})$ is much larger than its diameter $\left(D_{\text {core }} / D_{\text {clad }}=52 / 125 \mu \mathrm{m}\right)$, the effective emissivity is close to 1 . This result makes the dependence of the silica emissivity on wavelength and on temperature secondary issues in this particular case. The fitting of the fibre emission is shown in Fig. 7. A good agreement is obtained between the blackbody radiation and the experimental results.

\subsection{The arc discharge}

An arc discharge is a complex phenomenon during which a number of processes occur. In simple terms, it can be said that electrons are emitted by one electrode, the cathode, due to the high intensity electric field generated at the electrodes tip, and while in transit between the electrodes ionizes nitrogen and oxygen atoms, through impacts, creating a high temperature plasma comprising electrons and ions [12]. Fig. 8 shows the arc emission spectra as recorded by the PR-714 Photo Research spectroradiometer and by the Cronin spectrometer. For the former, the radiation during the arc discharge was detected by the spectroradiometer placed $\sim 2 \mathrm{~m}$ away from the splicing machine whilst focusing on the discharge area. For the latter, the arc radiation was detected by a multimode fibre placed in the vicinity of the arc. Similar non-normalized spectra were obtained for both arrangements.

\subsection{Fibre temperature}

The experimental set-up consists of a fusion splicing machine to produce the arc discharges, a piece of multimode

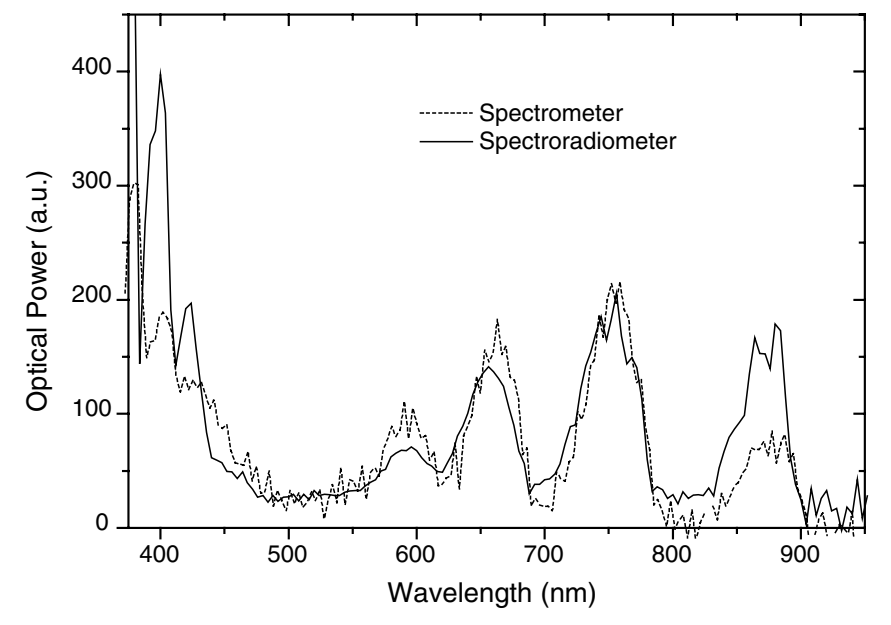

Fig. 8. Arcemission spectra recorded by the spectrometer/spectroradiometer.

fibre to generate and guide the radiation and a computer controlled Cronin spectrometer (Fig. 9). One end of a short piece of fibre $(l<0.5 \mathrm{~m})$ was placed between the electrodes of a splicing machine (electrodes gap $=1 \mathrm{~mm}$, tip angle $\sim 40^{\circ}$ ) and the other end was connected to the spectrometer. An electric arc discharge $(I=9 \mathrm{~mA}, t=3 \mathrm{~s})$ was then produced on the fibre, close to its end, and the radiation was detected by the spectrometer during one second after thermal equilibrium. The process was repeated for increasing electric arc currents up to $13 \mathrm{~mA}$. The registered spectra were corrected for the spectrometer response and fitted to the blackbody radiation expression over the $450-650 \mathrm{~nm}$ range, from which the fibre temperature $\left(T_{\mathrm{f}}\right)$ is obtained (Fig. 10). In this estimation, the fibre refractive index was set equal to 1.48 and it was considered that the fibre emissivity was independent of the wavelength, being its value set equal to 1. Fig. 11 shows the measured dependence of the fibre temperature with current. For an electric current of $9 \mathrm{~mA}$ an average temperature of $1365^{\circ} \mathrm{C}$ was determined. Note that for higher currents, the temperature dependence of the refractive index was not taken further into account and therefore it is possible that the slope of the linear fitting is higher than the value shown in Fig. 11. The dispersion of the values measured for the low and high current limits is probably related with the low level of the signal detected and the deformation of the fibre, respectively. It should be highlighted that the region heated by the arc has a non-uniform temperature distribution, and consequently the fibre emission spectrum is the sum of all contributions that result from dividing the heated region in smaller regions, each having a constant temperature. Therefore, the peak temperature values obtained for each arc current are underestimated. Detailed

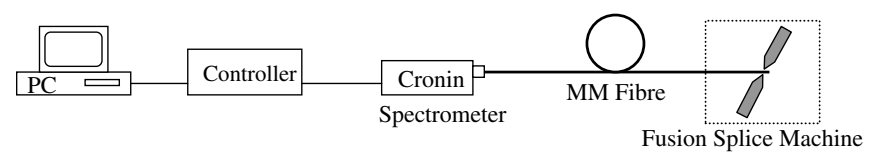

Fig. 9. Experimental set-up. 


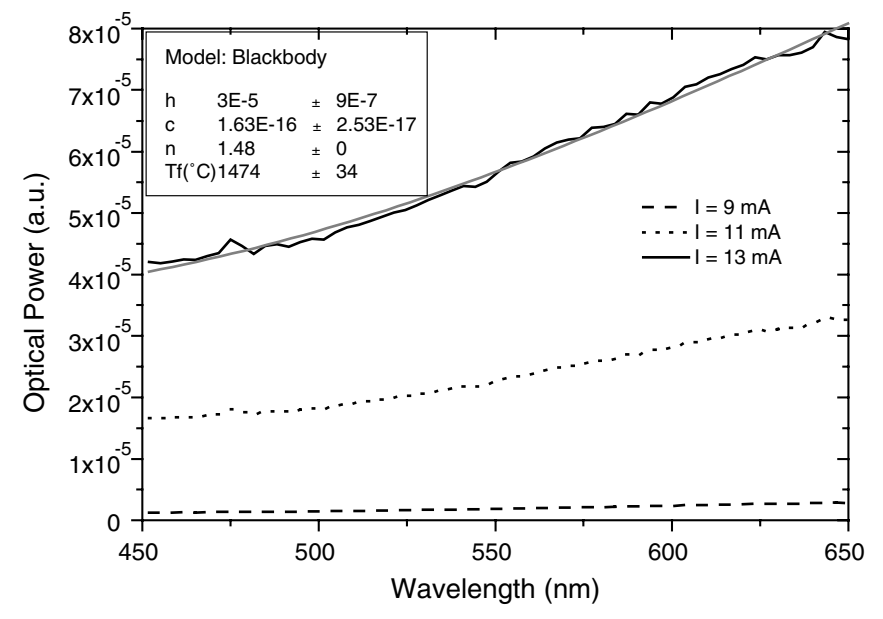

Fig. 10. Fitting blackbody radiation expression to the fibre emission spectra detected by the spectrometer. The grey line corresponds to the fitting of the fibre's emission at an arc current of $13 \mathrm{~mA}(h$ and $c$ are experimental constants resulting from optimization to the fitting).

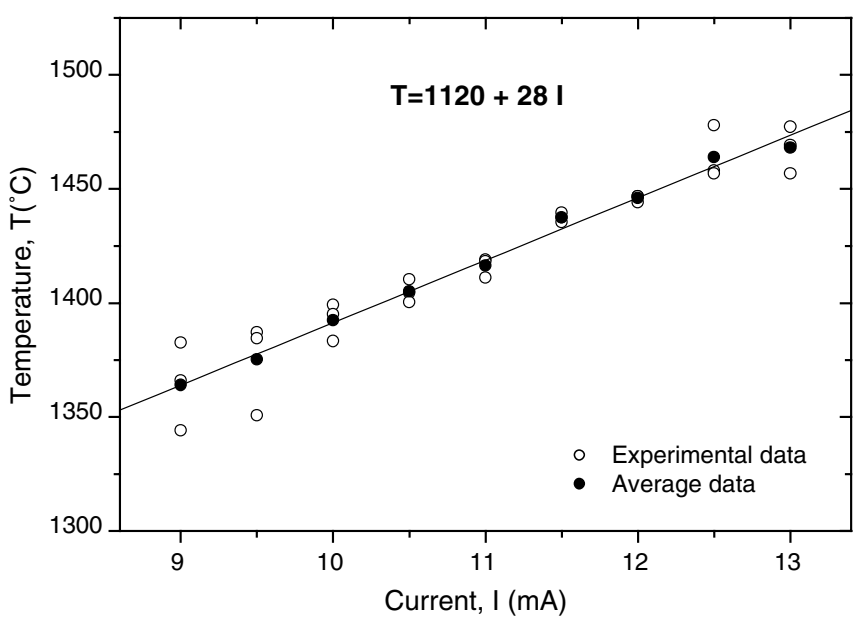

Fig. 11. Fibre temperature versus arc discharge current.

studies on non-isothermal cavities can be found in [13]. To have a better understanding of this effect, a particular fibre temperature profile having a peak temperature of $1320^{\circ} \mathrm{C}$ [14] was assumed and its division in 9 smaller regions considered. Taking into account that the silica emissivity increases considerably with temperature, only regions above $1150^{\circ} \mathrm{C}$ contribute significantly to the overall spectrum. U:/ES/DTD501/Optics/10881The silica emissivity, for a particular temperature, was estimated considering that it would be the same as the absorption in a length of $1 \mathrm{~mm}$, at the same temperature. Moreover, to determine the effective fibre emissivity, the ratio $L / D$ of the cavity was considered equal to $20(1000 / 50)$. It arose from the calculations that the emissivity at $1320^{\circ} \mathrm{C}$ was about 3 and 8 times higher than the one at 1230 and $1150^{\circ} \mathrm{C}$, respectively. Fig. 12 shows the blackbody emission spectrum of each region with temperatures above $1150^{\circ} \mathrm{C}$ and the overall spectrum. Fitting these curves to the blackbody radiation expression resulted in a temperature of $1280^{\circ} \mathrm{C}$. Therefore, taking into

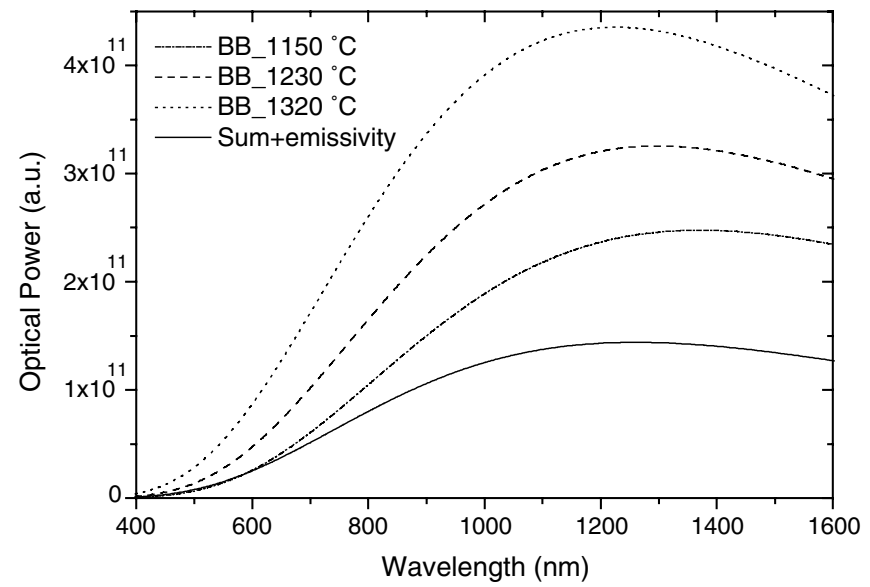

Fig. 12. Effect of having a non-uniform fibre temperature.

account the uncertainty in the refractive index $\left( \pm 40^{\circ} \mathrm{C}\right)$ and the temperature distribution $\left(\sim 40^{\circ} \mathrm{C}\right)$, the estimated peak value of the fibre temperature, correspondent to an arc discharge of $9 \mathrm{~mA}$, is $1400 \pm 50^{\circ} \mathrm{C}$.

\section{Conclusion}

A method based on the blackbody radiation model was presented to determine the temperature of a short piece of fibre exposed to an electric arc discharge. The radiation emitted by the fibre was first detected using a Cronin spectrometer and then its spectrum was fitted to the theoretical blackbody radiation. An average temperature of about $1365^{\circ} \mathrm{C}$ was obtained for the region exposed to an electric current of $9 \mathrm{~mA}$. However, a peak temperature of $1400 \pm 50{ }^{\circ} \mathrm{C}$ was estimated assuming a realistic temperature profile. It should be stressed that these calculations are strongly influenced by the thermal behaviour and the wavelength dependence of two physical parameters of the fibre, namely its refractive index and emissivity.

\section{Acknowledgements}

The authors acknowledge Prof. L.M. Bernardo and J.L. Costa for their collaboration. G.M. Rego is thankful for the grant conceded by the Program PRODEP III - Medida 5 - Acção 5.3. - Formação Avançada de Docentes do Ensino Superior, integrada no Eixo 3.

\section{References}

[1] A. Vengsarkar, P. Lemaire, J. Judkins, V. Bhatia, T. Erdogan, J. Sipe, J. Lightwave Technol. 14 (1996) 58.

[2] S.W. James, R.P. Tatam, Meas. Sci. Technol. 19 (2003) 49.

[3] O. Frazão, R. Romero, G. Rego, P.V.S. Marques, H.M. Salgado, J.L. Santos, Electron. Lett. 38 (2002) 693.

[4] G. Rego, O. Okhotnikov, E. Dianov, V. Sulimov, J. Lightwave Technol. 19 (2001) 1574.

[5] E.F. Zalewski, in: M. Bass (Ed.), Handbook of Optics, vol. II, McGraw-Hill, New York, 1995 (Chapters 24, 25).

[6] R. Bruckner, J. Non-Cryst. Solids 5 (1970) 123. 
[7] J.W. Fleming, in: Proceedings of the Optical Fiber Communications Conference, Los Angels, USA, TuB2, 2004.

[8] M.J. Adams, An Introduction to Optical Waveguides, Wiley, New York, 1981, p. 243.

[9] G. Keiser, Optical Fiber Communications, McGraw-Hill, New York, 2000 , p. 96.

[10] Y. Shuto, S. Yanagi, S. Asakawa, M. Kobayashi, R. Nagase, IEEE Photon. Technol. Lett. 16 (2004) 1008.
[11] W. Zhihai, C. Jiahua, Z. Hanyi, Z. Bingkun, J. Tsinghua Univ. 28 (s3) (1986) 31.

[12] J.D. Cobine, Gaseous Conductors, McGraw-Hill, New York, 1941, p. 290.

[13] V.I. Sapritsky, A.V. Prokhorov, Appl. Opt. 34 (1995) 5645.

[14] G. Rego, L.M.N.B.F. Santos, B. Schröder, P.V.S. Marques, J.L. Santos, H.M. Salgado, IEEE Photon. Technol. Lett. 16 (2004) 2111. 